Proc. Indian Acad. Sci. (Chem. Sci.), Vol. 106, No. 4, August 1994, pp. 903-911.

(C) Printed in India.

\title{
Distribution of ionic charge carriers and migration barriers in binary alkali silicate glasses
}

\author{
K J RAO* and S R ELLIOTT ${ }^{+}$ \\ Department of Chemistry, University of Cambridge, Lensfield Road, Cambridge CB2 1EW, \\ UK \\ * Permanent address: Solid State and Structural Chemistry Unit, Indian Institute of Science, \\ Bangalore 560012, India \\ +Also at: Laboratoire de Science des Materiaux Vitreux, Universite de Montpellier II, Place. \\ Eugene Bataillon, 34095 Montpellier Cedex, France
}

MS received 20 April 1994

\begin{abstract}
Likely spatial distributions of network-modifying (and mobile) cations in (oxide) glasses are discussed here. At very low modifier concentrations, the ions form dipoles with non-bridging oxygen centres while, at higher levels of modification, the cations tend to order as a result of Coulombic interactions. Activation energies for cation migration are calculated, assuming that the ions occupy (face-sharing) octahedral șites. It is found that conductivity activation energy decreases markedly with increasing modifier content, in agreement with experiment.
\end{abstract}

Keywords. Alkali ions; Silicate glasses; migration energy barriers; Spatial distribution; activation energies; non-bridging, oxygen ions.

\section{Introduction}

Migration barriers of alkali ions in silicate glasses have long been investigated experimentally by electrical conductivity and radioactive tracer self-diffusion measurements (Frischat 1975; Paul 1982). The activation energies are found to decrease rapidly with increasing concentration, $x$, of modifier oxide $\left(\mathrm{M}_{2} \mathrm{O}\right)$ in binary silicate glasses, $x \mathrm{M}_{2} \mathrm{O} \cdot(1-x) \mathrm{SiO}_{2}$ up to a concentration $x=0 \cdot 3$; thereafter, the activation energies tend to level off. This behaviour of the activation energy is found empirically to vary with composition in a similar manner to that of the average number of bridging oxygen (BO) atoms per Si atom, the so-called Stevels parameter (Paul 1982). Evidently, non-bridging oxygen (NBO) ions play an important role in determining the magnitudes of the activation barriers in modified silicate glasses.

Vitreous silica consists of a 3-D network of corner-shared $\left[\mathrm{SiO}_{4 / 2}\right]$ units and its structure has been investigated in a number of studies (Elliott 1990). Modification of the network can be visualized as a depolymerization process wherein $\mathrm{Si}-\mathrm{O}-\mathrm{Si}$ bonds associated with $\mathrm{BO}$ atoms are broken, with the production in pairs of $\mathrm{NBO}\left(\mathrm{Si}^{-} \mathrm{O}^{-}\right)$ centres when alkali oxides are added, viz.

$$
\mathrm{M}_{2}^{+} \mathrm{O}^{2-}+\mathrm{Si}-\mathrm{O}-\mathrm{Si} \rightarrow 2 \mathrm{M}^{+}+2\left[\mathrm{Si}-\mathrm{O}^{-}\right]
$$

\footnotetext{
* For correspondence
} 
Evidently there will be Coulombic interactions between the charged $\mathbf{M}^{+}$ions and the $\mathrm{NBO} \mathrm{O}^{-}$centres. In addition, $\mathrm{M}^{+}$ions polarize the significantly polarizable oxygen atoms (both $\mathrm{BO}$ and NBO), inducing strong dipoles on them. The interactions of the induced dipoles with the alkali ions themselves (the polarization self-energy) can be quite substantial at short distances. Furthermore, when the NBO's are present on neighbouring $\mathrm{SiO}_{4}$ tetrahedra, there is an electrostatic repulsion which tends to force them apart. Since the number of sites on which such NBO's can be located are numerous when the modifier content $x$ is small, it is entropically favourable for the NBO's to be randomly distributed.

Hence, in such materials, there are a number of significant and competing energies of interaction: the Coulombic attraction between $\mathrm{M}^{+}$and $\mathrm{NBO}$, the Coulombic repulsion between NBO's and between $\mathrm{M}^{+}$ions, the polarization self-energies of the $\mathrm{M}^{+}$ions and the entropy associated with the distribution of NBO's (T $S S$ terms). These determine the configuration of the ground state of a modifier cation in silicate melts (and hence in the quenched glasses). Anion polarization self-energies and overlap repulsions can be effectively neglected in this regard. Thus, if these energy terms are known, at least approximately, we can address the question of the variation of the energy barriers.

In this paper we show that, in dilute modified glasses, the NBO's tend to be separated from each other, but that the $\mathrm{M}^{+}-\mathrm{NBO}$ pairs tend to remain together. As the modifier content, $x$, increases, the mutual electrostatic interactions between different pairs of $\mathrm{M}^{+}$ions and $\mathrm{NBO}$ centres increase, and hence $\mathrm{M}^{+}-\mathrm{NBO}$ separations tend to increase so as preferentially to exploit the Coulombic interactions through a Madelung-type term. For such a situation, we show that the activation barriers for migration decrease with increasing $x$, by making the assumption that the alkali ions are located at the centres of face-sharing octahedra (comprising oxygen atoms), the faces in common thereby acting as "doorways", or short tunnels, for alkali-ion migration.

\section{Glasses with low modifier contents}

In modified glasses, several configurations of $\mathrm{M}^{+}$with respect to NBO sites can be envisaged. In cases when neighbouring NBO's do not separate, the lowest energy configuration would be as shown in figure 1 , comprising a square arrangement of two $\mathrm{M}^{+}$and two $\mathrm{NBO}$ atoms; the $\mathrm{M}^{+}$ions will also be coordinated by other $\mathrm{BO}$ atoms not shown in the figure. Evidently there will be a mutual Coulombic interaction between the four charged species but the polarization self-energy will be essentially zero since such close $\mathrm{M}^{+}-\mathrm{O}^{-}$pairs (effectively acting as dipoles) will polarize neighbouring BO atoms in the opposite directions. Thus, the energy of interaction can be written as:

$$
E_{1}=-x\left(\frac{4 e^{2}}{r}-\frac{2 e^{2}}{\sqrt{ } 2 r}\right)=-2 \cdot 6 \frac{x e^{2}}{r} .
$$

where $r$ is the $\mathrm{M}^{+}-\mathrm{O}^{-}$separation and $x$ is the mole fraction of modifier. (It should be noted that a tetrahedral arrangement of the four charged ions results in larger, less negative interaction energy, viz., $E_{1}=-2 \mathrm{xe}^{2} / r$ ). 


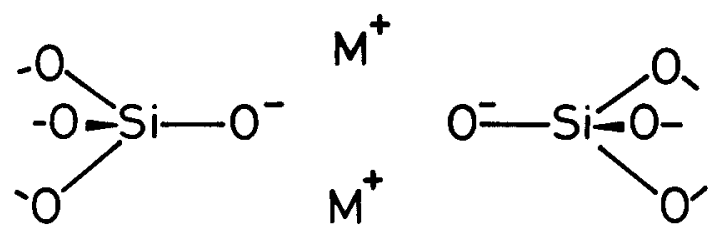

Figure 1. Schematic illustration of the depolymerization caused by the addition of networkmodifying cations, $\mathbf{M}^{+}$, to glassy silica. At low levels of modification, pairs of dipoles occur, formed from $\mathbf{M}^{+}$ions and non-bridging oxygen.

Let us now suppose that (in the melt prior to quenching to form the glass) the $\mathbf{M}^{+}-\mathrm{NBO}$ pairs drift away from each other (but each $\mathbf{M}^{+}$ion remains "bound" to an individual $\mathrm{NBO}$ site). Since a single $\mathrm{NBO}$ in an $\mathrm{SiO}_{4}$ tetrahedron can be written equivalently as a $Q_{3}$ unit, and bonds between tetrahedra can be switched such that

$$
Q_{3}+Q_{4} \rightarrow Q_{4}+Q_{3} \text {, }
$$

the $\mathrm{M}^{+}$ions in such isolated $\mathrm{M}^{+}-\mathrm{NBO}$ pairs can end up occupying energetically favourable sites in which the nearest-neighbour coordination shell comprises the single NBO and a number, $n$, of $\mathrm{BO}$ atoms. For the case of $\mathrm{Na}^{+}$, for example, $n=5-6$, and so the sites are essentially octahedral figure 2 . In such a case, the energy of interaction can be written as the sum of two terms, one Coulombic and the other a polarization term, viz.

$$
E_{2}=-\frac{2 x e^{2}}{r}-\frac{2 x \cdot 6 \alpha e^{2}}{r^{4}}=-\frac{2 x e^{2}}{r}\left[1+\frac{6 \alpha}{r^{3}}\right] .
$$

where the factor of 2 in both terms refers to the two $\mathrm{M}^{+}-\mathrm{NBO}$ configurations under consideration (one of which is shown in figure 2), and where $\alpha$ is the polarizability of the oxygen atoms (assumed to be the same for both BO and NBO configurations).

The configurational entropy associated with the separation of such $\mathrm{M}^{+}-\mathrm{NBO}$ pairs can be estimated as follows: For the composition $x \mathrm{M}_{2} \mathrm{O} \cdot(1-x) \mathrm{SiO}_{2}$, there are $N_{A}(1-x) \mathrm{SiO}_{4 / 2}$ tetrahedra (where $N_{\mathrm{A}}$ is the Avogadro number). Of these, $2 x N_{\mathrm{A}}$ tetrahedra are converted to $Q_{3}$ units by the incorporation of the modifier, therefore leaving $(1-3 x) N_{A}$ as $Q_{4}$ units. An upper limit to the value of the configurational energy can be obtained by simply considering permutations of this number of $Q_{3}$ and $Q_{4}$ units without any further constraints. (Restricting the number of permutations by precluding some configurations would result in a smaller value of the entropy.) In such a case, the number of complexion's arising from such a general permutation would be

$$
W=[1-x] ! /(1-3 x) !(2 x) !,
$$

and the resulting entropy would be (using Stirling's theorem):

$$
\Delta S=k \ln W=R[(1-x) \ln (1-x)-(1-3 x) \ln (1-3 x)-2 x \ln 2 x] .
$$

This term is maximal for $x=0.81$, and $\Delta S$ has the value of $0.56 \mathrm{JK}^{-1}$. The corresponding term, $T \Delta S$, will tend to stabilize the separated arrangement of the $\mathrm{M}^{+}-\mathrm{NBO}$ pairs in the melt. Taking the glass-transition temperature, $T_{\boldsymbol{\theta}}$, as the last temperature 


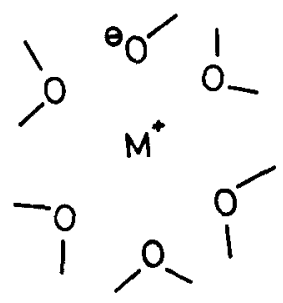

Figure 2. Schematic (two-dimensional) representation of an octahedral interstice containing a network-modifying cation, $\mathrm{M}^{+}$, formed from bridging oxygens and a nonbridging oxygen.

at which thermal equilibrium existed in the melt, and assuming, as usual, that this value also characterises the glassy phase, the largest value of $T \Delta S$ is $T_{g} \Delta S \approx 4.2 \mathrm{~kJ} / \mathrm{mol}$ or $0.04 \mathrm{eV}$, where $T=T_{g} \approx 900 \mathrm{~K}$ for alkali silicate glasses.

By contrast, we can compare this entropy term with the (Coulombic and polarization) energy terms, $E^{1}$ and $E^{2}$ [equations (1) and (2)] which are each of the order $3-6 \mathrm{eV}$; hence, the entropy term is completely negligible in determining whether or not $\mathrm{M}^{+}-\mathrm{NBO}$ pairs separate. The condition for separation of the pairs to be energetically favourable is when $E_{2}<E_{1}$, i.e. when $12 \alpha / r^{3}>0.6$. This condition is satisfied for the case, of sodium silicate glasses, if $\alpha$ (oxygen) $=0.5 \AA^{3}$ since $r \approx 2.3 \AA$. The value of $\alpha$ for $\mathrm{O}^{2-}$ ion has been reported to vary widely and it depends on the metal ion to which oxygen is covalently bonded. The value of $\alpha$ for an NBO should be lower than that of $\mathrm{O}^{2-}$ ion and close to that of a $\mathrm{BO}$. In view of the fact that $\mathrm{O}^{2-}$ has a polarizability of about $1.3 \AA^{3}$ we adopt a value of $1.0 \AA^{3}$ for polarizabilities of both $\mathrm{BO}$ and $\mathrm{NBO}$ in the present problem.

The condition determining the low-modifier-content limit is that the $Q_{3}$ units should be isolated, i.e. not in contact. A percolation path of contacting $Q_{3}$ species begins to be established at $x=0: 16$ (Elliott 1993), and so this marks the lowmodifier-content limit. Thus, for $x \leqslant 0 \cdot 16$, we expect that $\mathrm{M}^{+}-\mathrm{NBO}$ ion pairs always remain separated (but each pair remains bound together).

We have assumed that the only type of modified $\left[\mathrm{SiO}_{4 / 2}\right]$ units present in the glass are $Q_{3}$ configurations. Hence, equilibria of the type $2 Q_{3} \rightleftharpoons Q_{4}+Q_{2}$ have been neglected. We note in passing that such general equilibria between $Q_{n}$ species have been considered in detail by Gurman (1990), who postulates that the equilibrium (in the melt) is governed by the term $\exp [-\Delta E / k T]$ where the "bond-ordering energy" $\Delta E=E_{b b}+E_{n n}-2 E_{n b}$ and where $b$ and $n$ denote $\mathrm{BO}$ and NBO sites. respectively present in the same [ $\left.\mathrm{SiO}_{4}\right]$ tetrahedron. If the $\mathrm{BO}$ sites are assumed, for simplicity, to be uncharged, and the NBO sites carry a single negative charge, then $\Delta E \approx E_{n n} \sim$ $14.4 / r_{n n} \mathrm{eV}$, where $r_{n n}(\AA)$ is the intratetrahedral separation between NBO sites (in a $\mathrm{Q}_{2}$ configuration). Typically, $r_{n n} \approx 2.7 \stackrel{\circ}{A}$, and so $\Delta E \approx 5.4 \mathrm{eV}$, very much higher than the value for $\Delta E$ inferred by Gurman (1990) ' from a mass-action treatment of the relative proportion of $Q_{n}$ species derived from Raman and NMR data, viz $\Delta E \approx$ $2 k T_{g} \approx 0.2 \mathrm{eV}$, casting doubt on this approach. Gurman (1990) also made an estimate for $\Delta E$ based on electrostatic arguments similar to ours but the value he obtained $(\approx 0.25 \mathrm{eV})$ was arrived at by including the dielectric constant (a value corresponding to the bulk value, $\varepsilon \approx 10$ ). We regard this procedure as very dubious, since the charges on NBO sites in a $Q_{2}$ unit cannot be screened by the bulk dielectric constant at such 
short distances. Only by reducing the charge on an NBO to $0 \cdot 2 e$ can the magnitude of the electrostatic interaction be reduced to a value comparable to that deduced from mass-action considerations of bond-ordering energies.

\section{Glasses with higher modifier contents}

In the low modification regime $(x \rightarrow 0)$, there are several equivalent positions for an $\mathrm{M}^{+}$ion around an NBO site for which the energy of the system is effectively the same. However, at high modifier contents, each $\mathbf{M}^{+}$ion interacts (Coulombically) with several other ions and its equilibrium position therefore corresponds to a position of minimum Coulombic energy. Thus, when it moves (adiabatically) out of such a position, the interaction energies are expected to be altered drastically. The vacated position is quite similar to that of a Schottky defect in crystalline solids. Indeed, the imbalance of charge (virtual charge of opposite sign) at the "vacancy" when an ion is not at the equilibrium position causes polarization and, hence, results in a polarization energy if the surrounding ions do not readjust their positions correspondingly.

\section{Migration energies in the low- and high-modification regimes}

Recent EXAFS analyses of the structure of alkali silicates suggest that, except for $\mathrm{Li}^{+}$ions, the alkali ions occupy sites in which they are coordinated by 5-6 oxygen atoms (Greaves et al 1991); six-fold coordination of $\mathrm{Na}^{+}$ions in glasses is perhaps the most frequently observed. We further assume that the local geometry of the sites is octahedral. The triangular faces of such octahedra could be connected to similar octahedra and/or to tetrahedra. Although a space-filling arrangement of all-facesharing octahedra is not possible, it is possible to envisage an arrangement where an octahedron shares faces with two other octahedra, thereby forming a (zig-zag) chain of connected octahedra. When the modifier content is large $(x>0.16)$, there is, on average, one NBO per octahedron. As a result, the path involving face-sharing octahedra becomes decorated with NBO sites forming a percolation path of connected NBO sites (Elliott 1993). This picture is somewhat reminiscent of the "channels" in the modified random network (MRN) picture of Greaves (1985), except that here we are more specific about the coordination polyhedra, and their connections, to the network modifier sites.

The principal motif is a pair of face-sharing octahedra, which we may consider first to contain an $\mathrm{NBO}$ site and an $\mathrm{Na}^{+}$ion. This arrangement is a generalization of the model involving dioctahedra containing only non-bridging sites considered for the case of highly modified lithium thioborate glasses by Rao et al (1993). The simplest representation of two-face-sharing octahedra is as three equilateral triangles in parallel ABAB stacks, with their centres aligned to form an axis (see figure 3). Viewed along the axis, this arrangement has cylindrical symmetry which permits easy evaluation of all relevant distances. Let us suppose that one of the oxygen atoms in the dioctahedron is an NBO and consequently the remaining eight oxygen atoms are $\mathrm{BO}$ configurations. If this situation represents the overall average composition, then the ratio of NBO sites to $\mathrm{BO}$ sites is $1 / 8=2 x /(2-3 x)$, corresponding to the modifier content $x=0.11$ which is within the low-modification regime. 


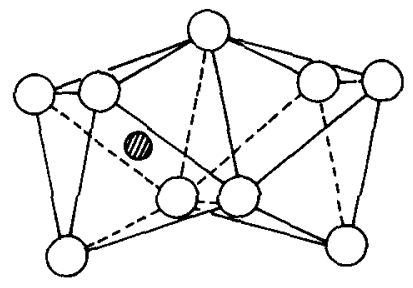

a)

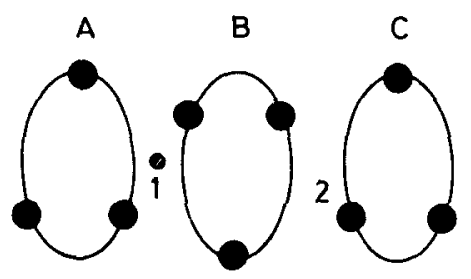

b)

Figure 3. Two face-sharing octahedral interstices (a) and an equivalent representation of the dioctahedron (b) emphasizing the local cylindrical symmetry of such a unit In (b) the oxygen ligands lie on circles $\mathrm{A}, \mathrm{B}, \mathrm{C}$ and the stable positions of the cation $\mathrm{M}^{+}$are between such rings.

The NBO can be present on any of the three rings shown in figure 3. Let us first suppose that the $\mathrm{NBO}$ is on ring $\mathrm{A}$ (or $\mathrm{C}$ ) and that the ion $\mathrm{M}^{+}$is at position 1 (or 2 ). We consider that the primary event of migration is the motion of the $\mathbf{M}^{+}$ion from position 1 (or 2 ) to 2 (or 1 ). When the ion moves through the migration doorway which is the middle ring comprising three $\mathrm{BO}$ atoms, it displaces these atoms radially outwards. These displaced atoms, in turn, exert an outward compression on the rest of the structure through the covalent bonds connecting into the network. This is the source of the so-called strain energy (Chan and Elliott 1991). For the purpose of this calculation, we assume that all the octahedra are regular, and consequently, from the geometry of octahedra, when the $\mathrm{M}^{+}$ion is at the centre of the central ring (the activated saddle-point position), the BO atoms on the ring are displaced by a distance of $0.18 r$ (where $r$ is the normal $\mathrm{M}^{+}-\mathrm{BO}$ or $\mathrm{M}^{+}-\mathrm{NBO}$ distance) so that the $\mathrm{M}^{+}-\mathrm{BO}$ distance in the saddle-point position is also $r$.

Let the energy of the $\mathrm{M}^{+}$ion in the ground state (position 1 or 2) be $E_{G}$ and in the activated saddle-point state be $E_{S}$. The activation barrier is therefore $\Delta E=E_{S}-$ $E_{G}$. If we consider Coulombic, polarization and overlap repulsion terms only, neglecting the strain-energy term which is very small, $\leqslant 0.1 \mathrm{eV}$ (Rao et al 1993; Elliott 1993), and if we use an exponential form of the repulsion energy (Rao et al 1993), then

and

$$
E_{\sigma}=-\frac{e^{2}}{r}-\frac{6 \alpha e^{2}}{r^{4}}-\frac{3 \alpha e^{2}}{\left((11 / 3 r)^{1 / 2}\right)^{4}}+6 b \exp (-r / \rho)
$$

$$
E_{s}=-\frac{e^{2}}{\sqrt{2 r}}-\frac{3 \alpha e^{2}}{r^{4}}-\frac{6 \alpha e^{2}}{(\sqrt{ } 2 r)^{4}}+3 b \exp (-r / \rho),
$$

where again we have assumed that the polarizabilities $\alpha$ of both $\mathrm{BO}$ and NBO species are the same. Thus, the migration activation energy is:

$$
\Delta E \approx 0 \cdot 29 e^{2} / r+1 \cdot 72 \alpha e^{2} / r^{4}-3 b \exp (-r / \rho) .
$$

Hence the activation energy consists of positive Coulombic and polarization terms and a negative repulsive contribution. In the situation pictured in figure 3 , the motion of the ion is in a direction opposite to that of the Coulombic attraction.

The individual terms in (7) can be evaluated as follows. The Coulombic term is 
equal to $1.82 \mathrm{eV}$ if the $\mathrm{Na}^{+}-\mathrm{O}^{-}$distance is taken to be $r=2.3 \AA$. If the polarizability of oxygen atoms (BO and $\mathrm{NBO}$ ) is $1.0 \AA^{3}$, the polarization term contributes $0.88 \mathrm{eV}$. The repulsive contribution can be estimated, as is commonly done for ionic systems, by assuming that the net repulsive energy in the ground state is approximately $20 \%$ of the total ionic cohesive energy (Rao et al 1993), viz

$$
6 b \exp (-r / \rho)=0 \cdot 2 \alpha_{M} e^{2} / r,
$$

where $\alpha_{M}$ is an appropriate Madelung constant. If $\alpha_{M}$ is taken to have the value $=1.7$ (Rao et al 1993), then the net repulsive term in (7) is $\Delta E^{R}=0.17 e^{2} / r=1.06 \mathrm{eV}$ for the case of $\mathrm{Na}^{+}-\mathrm{O}^{-}$interactions. Thus, the total (conductivity) activation energy is estimated from (7) to be $\Delta E=1.66 \mathrm{eV}$. This estimate compares well with experimental values of the ionic d.c. conductivity activation energy for low-modifier-content oxide glasses, for which $E_{\sigma} \leqslant 1.7 \mathrm{eV}$ (Frischat 1975; Martin 1988). However, there are uncertainties related to the polarization term because of the corresponding uncertainty in the value of the polarizability, $\alpha$, of the oxygen species. Uncertainties are also present in the repulsive and Coulombic terms due to the nature of assumptions made about their relation and also due to the assumption of the value of the Madelung constant.

At the beginning of the high-modification regime, we would expect more NBO centres to be present and possibly also a more extended structure consisting of face-sharing octahedra. We consider one such configuration in figure 4 , where three dioctahedra share mutual faces. The three $\mathbf{M}^{+}$ions associated with this unit are located at the positions marked + and the three NBO sites at the positions denoted by - . The dotted lines indicate the resemblance to a "unit cell" in this representation, although we should remember that such face-sharing octahedra actually form contorted chains with no unique axis of propagation. The dots in figure 4 without associated labels are $\mathrm{BO}$ atoms. The composition of the cluster thus comprises $3 \mathrm{NBO}$ and $15 \mathrm{BO}$ atoms or $2 x /(2-3 x)=3 / 15$, corresponding to a modifier content $x=0.15$ which marks the beginning of the high-modification regime.

When the central $\mathrm{M}^{+}$ion moves to the position between rings 4 and 5 , it is in a similar site to where it was before the migration step because it is still in the vicinity of an NBO atom at a distance r. However, the energy would be slightly different

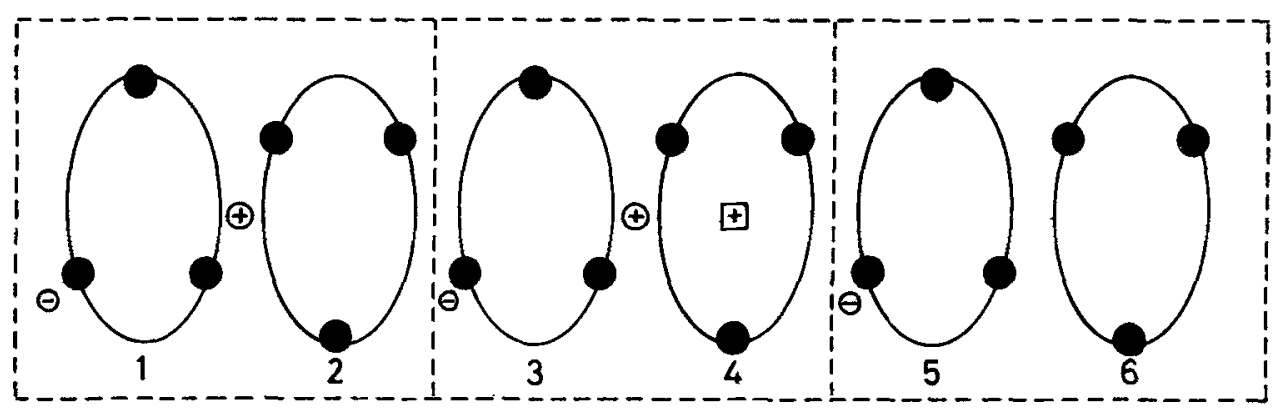

Figure 4. A schematic representation of three face-sharing dioctahedra. Filled circles on the rings represent oxygen atoms; those denoted by $\theta$ are non-bridging sites. Stable positions for the cations $\mathrm{M}^{+}$are denoted by $\oplus$ and a metastable, saddle-point position at the centre of a common face is denoted by $\boxplus$. Although this representation implies that such linked dioctahedra form straight chains, in practice such chains are contorted. 
because of the influence of the other cation. With the same notations as used before, we will calculate $E_{\mathrm{G}}$ and $E_{\mathrm{S}}$ (the superscripts $\mathrm{C}, \mathrm{P}$ and $\mathrm{R}$ refer to Coulombic, polarization and repulsion terms, repectively). Thus,

and

$$
E_{G}^{c}=-\frac{e^{2}}{r}-\frac{e^{2}}{r(11 / 3)^{1 / 2}}-\frac{e^{2}}{r(27 / 3)^{1 / 2}}+\frac{2 e^{2}}{4 r / 3^{1 / 2}}=-\frac{0.99 e^{2}}{r}
$$

Similarly

$$
E_{G}^{S}=-\frac{e^{2}}{r \sqrt{ } 2}-\frac{e^{2}}{r \sqrt{ } 2}-\frac{e^{2}}{r(38 / 3)^{1 / 2}}+\frac{e^{2}}{r\left(3 / 3^{1 / 2}\right)}+\frac{e^{2}}{r\left(5 / 3^{1 / 2}\right)}=-\frac{0.77 e^{2}}{r}
$$

and

$$
E_{G}^{P}=-\frac{6 \alpha e^{2}}{r^{4}}-\frac{6 \alpha e^{2}}{\left[r(11 / 3)^{1 / 2}\right]^{4}}-\frac{6 \alpha e^{2}}{\left[r(27 / 3)^{1 / 2}\right]^{4}}=-\frac{0.54 e^{2}}{r}
$$

$$
E_{s}^{P}=-\frac{3 \alpha e^{2}}{r^{4}}-\frac{6 \alpha e^{2}}{(r \sqrt{ } 2)^{4}}-\frac{6 \alpha e^{2}}{\left[r 6^{1 / 2}\right]^{4}}-\frac{3 \alpha e^{2}}{\left[r(38 / 3)^{1 / 2}\right]^{4}}=-\frac{0.38 e^{2}}{r},
$$

where again a value of $\alpha=1.0 \AA^{3}$ has been used. As before

and

$$
E_{G}^{R}=6 b \exp (-r / \rho)
$$

$$
E_{s}^{R}=3 b \exp (-r / \rho),
$$

and we take the relative value of the repulsive energy as given in (8). Thus, $E=\Delta E^{\mathrm{c}}+$ $\Delta E^{P}+\Delta E^{R}=0.21 e^{2} / r=1.26 \mathrm{eV}$. Thus, a marked decrease is predicted in the value of the ionic migration energy from the value characteristic of low concentrations of network modifiers. The ionic d.c. conductivity activation energy decreases to plateau values in the range $0.6-0.8 \mathrm{eV}$ in oxide glasses when modifier content increases to values $x=0.2-0.3$ (Frischat 1975; Martin 1988). However, in addition to the uncertainties in the theoretical estimate, there are uncertainties in values of the numerical constants like the polarizability. Equations (11) and (12), may also be erroneous, since the last term in $E_{G}^{P},(11)$, and the last two terms in $E_{s}^{P},(12)$, are affected by the polarization of other cations.

We expect the Coulombic and polarization energy contributions to vary rather nonlinearly as the modifier ion content $x$ increases. However, in the absence of detailed structural models in which the positions of cations and non-bridging anion sites are known (as a function of $x$ ), it would be inappropriate to speculate on the likely behaviour of $E_{\sigma}$ as a function of composition between the two representative values estimated above. It is interesting to note here that in the low alkali regime the ratio of Coulombic to polarization energy was $1 \cdot 8 / 0.88 \approx 2.0$ and it decreased to $22 / 16 \approx 1.4$, thereby suggesting that the polarization energy term becomes increasingly more important at higher levels of modification. A somewhat similar conclusion was arrived at by Elliott (1993) independently; activation energy at low modifier contents depends primarily on the Coulombic term, whereas at higher levels of modification, the smaller polarization term becomes dominant. 


\section{Conclusions}

It has been shown that the structural environment of network-modifying cations, $\mathrm{M}^{+}$, in glasses could be markedly different in two composition regimes: (1) in the low-modifier-content regime, ion pairs $\left(\mathrm{M}^{+}\right.$-non-bridging anion) are formed and occur at well-separated distances; (2) at higher modifier contents, the ion pairs are further separated and tend to order for taking advantage of Madelung-type Coulombic energy terms. It has also been demonstrated that modifier-ion clustering does not occur until very high levels of modification are reached.

Activation barriers for $\mathrm{Na}^{+}$ion migration in oxide glasses have been calculated for two representative values of modifier content $(x=0.11$ and $0 \cdot 15)$, corresponding respectively to the low-modification regime and the beginning of the high modification regime. Assuming that the alkali ions occupy (face-sharing) octahedral sites, coordinated by bridging and non-bridging oxygen species. These calculations show that the (ionic conductivity) activation energy decreases markedly with increasing modifier content, in conformity with experimental observation.

\section{Acknowledgements}

KJR wishes to thank the British Council (Indian Office) and the Royal Society of London for financial support which enabled him to visit the Department of Chemistry, University of Cambridge. SRE is grateful to the Nuffield Foundation for a Science Research Fellowship.

\section{References}

Chan S L and Elliott S R 1991 J. Phys. Condens. Matter 31269

Elliott S R 1990 Physics of amorphous materials 2nd edn (London: Longman)

Elliott S R 1993 J. Non-Cryst. Solids 16029

Frischat G H 1975 Ionic diffusion in oxide glasses (New York: Trans Tech)

Greaves G N 1985 J. Non-Cryst. Solids 71203

Greaves G N, Gurman S J, Catlow C R A, Chadwick A V, Houde-Walter S, Henderson C M B and Dobson B R 1991 Philos. Mag. A64 1059

Gurman S J 1990 J. Non-Cryst. Solids 125151

Martin S W 1988 J. Am. Ceram. Soc. 71438

Paul A 1982 Chemistry of glasses (New York: Chapman and Hall)

Rao K J, Estournes C, Levasseur A, Shastry M C R and Menetrier M 1993 Philos. Mag. B67 389

Shastry M C R and Rao K J 1989 Solid State Ionics 3717 